\title{
Chemokine expression in patients with ovarian cancer or benign ovarian tumors
}

Marek Nowak ${ }^{1}$, Łukasz Janas ${ }^{1}$, Malwina Soja ${ }^{1}$, Ewa Głowacka ${ }^{2}$, Krzysztof Szyłło ${ }^{1}$, Marcin Misiek ${ }^{3}$, Magdalena Klink ${ }^{4}$

\author{
${ }^{1}$ Department of Operative Gynecology and Gynecologic Oncology, Polish Mother's \\ Memorial Hospital - Research Institute, Lodz, Poland \\ '2aboratory Diagnostics Centre, Polish Mother's Memorial Hospital - Research \\ Institute, Lodz, Poland \\ ${ }^{3}$ Department of Gynecology, Holy Cross Cancer Centre, Kielce, Poland \\ ${ }^{4}$ Institute of Medical Biology, Polish Academy of Sciences, Lodz, Poland
}

Submitted: 9 May 2019; Accepted: 12 July 2019

Online publication: 21 March 2021

Arch Med Sci 2022; 18 (3): 682-689

DOI: https://doi.org/10.5114/aoms/110672

Copyright $\odot 2022$ Termedia \& Banach

\section{Abstract}

Introduction: Chemokines play a crucial role in tumor growth and progression according to proangiogenic and immunosuppressive action. The aim of this study was to investigate the serum levels of selected chemokines in patients with ovarian cancer or benign ovarian tumors to assess their role in tumorigenesis and their potential use in preoperative diagnosis of an adnexal mass.

Material and methods: The study group consisted of 59 women with ovarian cancer: 17 epithelial ovarian cancer (EOC) patients and 42 women with benign ovarian tumors. We measured in sera obtained preoperatively the level of CA125 and a panel of 5 chemokines - CX3CL1/fractalkine, CXCL1/ GRO- $\alpha$, CXCL12/SDF-1, CCL20/MIP-3 $\alpha$ and IL-17F - using the chemiluminescence method with multiplexed bead based immunoassay.

Results: CX3CL1 was significantly elevated in sera of advanced ovarian cancer patients compared to women with benign ovarian tumors. The significant elevation of CXCL1 was also observed (both early and advanced stages). A similar pattern was present with the standard ovarian cancer marker CA125. In our patients with endometriotic cysts CA125 levels were significantly higher than in women with other benign tumors, whereas all analyzed chemokines had similar serum titers in patients with endometriotic vs. other benign ovarian cysts.

Conclusions: CX3CL1 and CXCL1 are elevated in sera of EOC patients, which indicates their role in cancer development. Moreover, they might be useful in preoperative differential diagnosis of ovarian tumors, especially as they were not elevated in cases of endometriosis.

Key words: ovarian cancer, endometriosis, CX3CL1, CXCL1.

\section{Introduction}

Chemokines, in other words chemotactic cytokines, primarily regulate the motility and migratory properties of cells [1]. In the tumor microenvironment, they play a crucial role in the tumor cells' survival, growth and proliferation. It is also known that they strongly participate in tumor progression according to their proangiogenic and prometastatic action. Moreover, chemokines have a confirmed modulatory effect on the

\author{
Corresponding author: \\ Marek Nowak MD, PhD \\ Department of \\ Operative Gynecology \\ and Gynecologic \\ Oncology \\ Polish Mother's \\ Memorial Hospital - \\ Research Institute \\ 281/289 Rzgowska St \\ 93-338 Lodz, Poland \\ Phone: +48 422711159 \\ E-mail: mrn@poczta.onet.pl
}


immune cells' activity, both present in the tumor microenvironment and circulating in peripheral blood. Among others they play a crucial role in leukocyte infiltration into the tumor; therefore they control what type of effector cells $\left(\mathrm{CD}^{+}, \mathrm{CD} 8^{+}\right.$, macrophages, NK cells) are required [2-4]. Among many chemokines that are involved in cancer progression, not widely investigated in patients with ovarian cancer, those mentioned below are worth distinguishing.

CX3CL1, or fractalkine, is the only member of the $\mathrm{CX} 3 \mathrm{C}$ subfamily of chemokines and exists in two forms. The membrane-anchored form mediates strong cell adhesion while the soluble form acts mainly as a chemoattractant for T cells, NK cells, dendritic cells and CD14 positive monocytes [5-7]. CX3CL1 is a specific ligand of the CX3CR1 receptor [8]. The expression of $\mathrm{CX} 3 \mathrm{CL} 1$ and $\mathrm{CX}$ $3 C R 1$ has been established in different cancers such as breast, prostate, pancreatic and ovarian cancer [9-13].

CXCL12, also known as stromal-derived factor $1 \alpha$ (SDF-1 $\alpha)$, is a chemokine member of the angiogenic CXC family strongly associated with tumor growth, metastasis, angiogenesis, and chemoresistance. Moreover, it also plays an important role in the communication between tumor cells and their stromal cells in the tumor microenvironment. CXCL12 and its receptor CXCR4 have been found to be overexpressed in a variety of solid tumors: breast, colon, prostate, melanoma, and ovarian cancer [4, 14-16].

CXCL1, also called growth regulated oncogene- $\alpha$ (GRO- $\alpha$ ), another member of the CXC family, interacts with CXCR2. This protein is best known as an effective chemoattractant guiding neutrophils through venular walls. However, its involvement in tumor cell proliferation and migration as well as cancer progression, although less known, was also described. GRO- $\alpha$ was found to be expressed at a high level in a series of human cancers such as breast, gastric, bladder and ovarian $[6,17,18]$. GRO- $\alpha$ together with CXCL8 causes robust endothelial cell proliferation, tube formation and migration in a mouse model of ovarian cancer [19].

CCL20 is a small protein, with CCR6 as its cellular receptor [20]. Previous studies demonstrated that TNF activates the nuclear factor- $\kappa \mathrm{B}$ signaling to induce proinflammatory chemokines, CCL20 being one of them [21]. Serum concentration of CCL20 may be elevated in women with ovarian cancer [22].

IL-17F is a member of the IL-17 family, mainly produced by T-helper (Th17) cells and macrophages, sharing the strongest amino acid sequence with IL-17A. II-17F participates in allergic inflammation in the lung, and intestinal inflammation in vivo [23].
Ovarian cancer is still the leading problem in gynecological oncology. It accounts for about $25 \%$ of gynecological malignant neoplasms, but is responsible for almost $50 \%$ of deaths caused by these malignancies. Almost $75 \%$ of patients are diagnosed in the advanced stage of the disease (stages III and IV) due to the absence or nonspecific clinical symptoms at the beginning and the lack of screening methods for diagnosis. The gold standard in the diagnosis of pelvic masses is still a bimanual gynecological examination supplemented by transvaginal sonography and serum markers. The most common serum marker used is CA125. However, it is not specific for this disease. It is elevated in about $80 \%$ of patients with ovarian cancer, but also in about $60-80 \%$ of cases of liver cirrhosis, up to $20 \%$ of patients with endometriosis, benign and borderline ovarian tumors and in $1-5 \%$ of healthy women [24]. In the last few decades, many serologic biomarkers have been evaluated during the diagnosis of ovarian cancer (e.g. human epididymis protein 4 (HE4), soluble mesothelin-related peptides (SMRP), activin, inhibin, osteopontin, leptin, IL-6, IL-8), including those based on tumor-host immunologic interactions (various cytokines and antibodies) [24]. Up till now only HE4 (ROMA) has started to be applied in clinical practice.

This study aimed to investigate the serum levels of selected cytokines/chemokines in patients with ovarian cancer or benign ovarian tumors and their potential use in preoperative diagnosis of adnexal masses.

\section{Material and methods}

\section{Patients}

Our study, approved by the local Ethical Committee, was carried out in the Department of Operative Gynecology and Gynecologic Oncology of the Polish Mother's Memorial Hospital - Research Institute, Lodz, Poland. We checked the patients with a pelvic mass suspected to be an ovarian tumor admitted to the department. Patients with a history of any previous malignant neoplasia or gynecologic operation, transplantation, autoimmunologic disease, diabetes, thyroid problems or any signs of infection were excluded from the study. After operative treatment and histopathologic examination, we finally collected 59 patients for further analysis: 17 women with epithelial ovarian cancer (mean age: 60.4 years; range: 37-82 years) and 42 age-matched women with benign ovarian tumors of epithelial origin (mean age: 54.2 years; range: $30-80$ ). The stage of ovarian cancer was established after laparotomy and pathologic examination, according to the International Federation of Gynecology and Obstetrics 
Table I. Ovarian cancer patients' characteristics $(n=17)$

\begin{tabular}{|lcc|}
\hline Parameter & $\boldsymbol{N}$ & $\%$ \\
\hline Age (mean \pm SD) & $60.4 \pm 12.4$ & \\
\hline FIGO stage: & 7 & 41 \\
\hline I/II & 10 & 59 \\
\hline III/IV & & \\
\hline Histology: & 9 & 53 \\
\hline Serous & 3 & 18 \\
\hline Mucinous & 1 & 6 \\
\hline Endometrioid & 2 & 12 \\
\hline Clear cell & 1 & 6 \\
\hline Undifferentiated & 1 & 6 \\
\hline Brenner & 8 & 18 \\
\hline Grade: & 3 & 35 \\
\hline 1 & 67 \\
\hline 2 & 8 & \\
\hline 3 & 1 & \\
\hline
\end{tabular}

Table II. Patients with benign ovarian tumors $(n=42)$

\begin{tabular}{|lcc|}
\hline Parameter & $\boldsymbol{N}$ & $\%$ \\
\hline Age (mean \pm SD) & $54.2 \pm 11.9$ & \\
\hline Histology: & & \\
\hline Endometrial & 12 & 29 \\
\hline \begin{tabular}{l} 
Others: \\
\cline { 2 - 3 } Serous
\end{tabular} & 30 & 71 \\
\hline Mucinous & 19 & 45 \\
\hline Simple cysts & 7 & 17 \\
\hline
\end{tabular}

(FIGO) classification and protocol [25]. All tissues removed during surgery were examined by a pathologist: tumor grade, histological type and the presence of metastases were established, according to FIGO and WHO classifications [25, 26].

The clinical and histopathological data of ovarian cancer patients are listed in Table I. In our study group, 7 (41\%) women were diagnosed and operated on in the early stage of the disease (stages $\mathrm{I}$ and II) and 10 (59\%) patients were suffering from advanced ovarian cancer (stages III and IV). The most common was serous (53\%), then mucinous (18\%) and clear cell carcinoma (12\%). The mean age of ovarian cancer patients and women with benign ovarian tumors did not differ significantly $(60.4 \pm 12.4$ vs. $54.2 \pm 11.9)$. The benign ovarian tumors were mainly serous (45\%), endometrial (29\%) and mucinous (17\%) (Table II).

\section{Cytokine/chemokine determinations}

Peripheral venous blood from patients with ovarian tumors was obtained in the morning of the day of the surgery and transported to the lab- oratory within $1 \mathrm{~h}$ of collection. Body fluid samples were centrifugated at $1500 \times \mathrm{g}$ for $10 \mathrm{~min}$ and then stored at $-80^{\circ} \mathrm{C}$ until analyzed. We measured a panel of 5 cytokines/chemokines CX3CL1 (fractalkine), CXCL1 (GRO- $\alpha), \mathrm{CXCL} 12$ (SDF-1), CCL20 (MIP-3 $\alpha$ ), and IL-17F - using the chemiluminescence method with multiplexed bead based immunoassay (Panomics, Inc., USA), which allowed simultaneous analysis of numerous analytes in one sample. The results are presented in $\mathrm{pg} / \mathrm{ml}$. The CA125 level in the blood samples was measured using Elecsys CA125 II assay (Roche Diagnostics, Indianapolis, USA), in accordance with the manufacturer's instructions and expressed in $\mathrm{U} / \mathrm{ml}$.

\section{Statistical analysis}

For the continuous data we calculated mean, median, standard deviation (SD) and standard error of the mean (SEM). Verification of the distributions was made with the Shapiro-Wilk test. To compare the differences among groups we used the Mann-Whitney $U$ test. The differences were considered statistically significant for $p$-value $<0.05$.

\section{Results}

CX3CL1 (fractalkine) was significantly elevated in sera of ovarian cancer patients compared to women with benign ovarian tumors ( $13.3 \pm 7.9 \mathrm{pg} /$ $\mathrm{ml}$ vs. $6.7 \pm 3.0 \mathrm{pg} / \mathrm{ml}, p<0.005)$. When FIGO stage was taken into consideration we found that the mean level of serum CX3CL1 in advanced ovarian cancer (FIGO III/IV) was $15.3 \pm 8.7 \mathrm{pg} / \mathrm{ml}$ and was significantly higher than in the group of benign ovarian cysts $(p<0.01)$; in the early ovarian cancer group (FIGO I/II) it was $10.5 \pm 5.7 \mathrm{pg} / \mathrm{ml}$ and did not differ significantly from the benign group (Figure 1).

The levels of CXCL1 (GRO- $\alpha)$ were also significantly higher in sera of patients with ovarian cancer than in the benign group $(36.5 \pm 18.4 \mathrm{pg} / \mathrm{ml} \mathrm{vs}$. $17.6 \pm 8.7 \mathrm{pg} / \mathrm{ml}, p<0.01)$. Moreover, both early and advanced ovarian cancer patients (FIGO I/II: $27.5 \pm 8.6 \mathrm{pg} / \mathrm{ml}$; FIGO III/IV: $42.7 \pm 20.6 \mathrm{pg} / \mathrm{ml}$ ) had significantly higher levels of CXCL1 than women with benign ovarian tumors $(p<0.05)$ (Figure 1 ). The same differences were observed in the levels of the standard ovarian cancer serum marker CA125; in both groups of ovarian cancer patients its amount was significantly elevated (Figure 1).

Serum concentrations of CXCL12 (SDF-1) were similar in all investigated groups of patients (Figure 1). Only 2 (3.4\%) patients had serum titers of CCL20 (MIP-3 $\alpha$ ) and 12 (20.3\%) of IL-17F over the lower limit of detection $(2.0 \mathrm{pg} / \mathrm{ml}$ and $1.06 \mathrm{pg} / \mathrm{dl}$, respectively), so these cytokines were excluded from the analysis. 
A

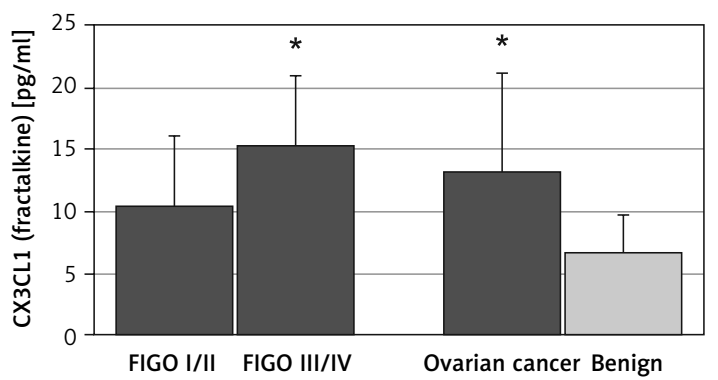

C

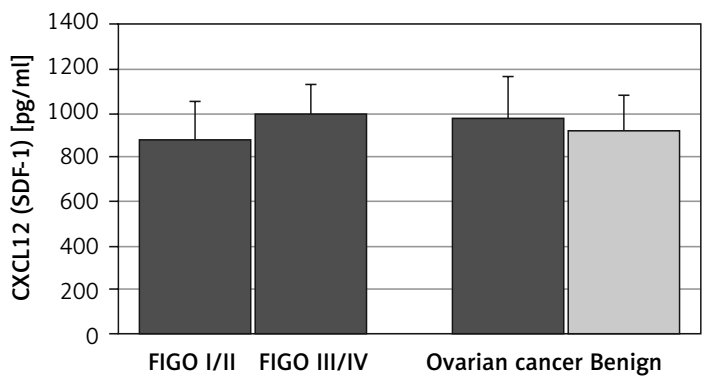

B

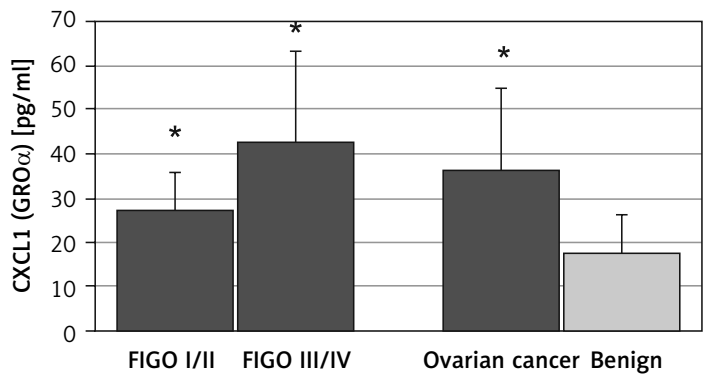

D

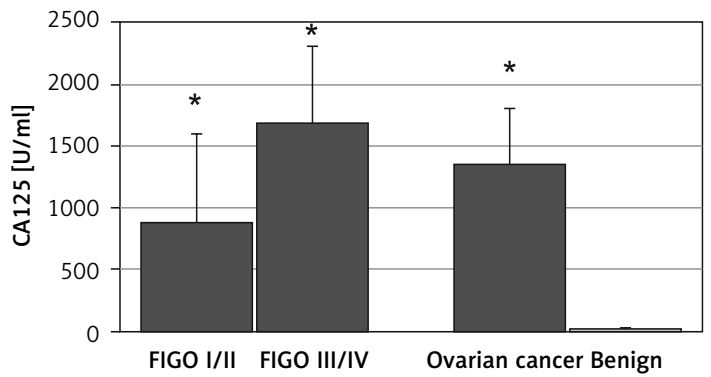

Figure 1. Serum concentrations of selected chemokines and CA125 in patients with ovarian cancer or benign ovarian tumors. Data are presented as a means $\mathrm{pg} / \mathrm{ml} \pm \mathrm{SD}$ (CA125 as means $\mathrm{U} / \mathrm{ml} \pm \mathrm{SEM}$ ). Statistical significance:

*early, advanced or overall ovarian cancer vs. benign ovarian tumors, $p \leq 0.05$

The main limitation of CA125 is that its serum concentration is elevated not only in cases of ovarian cancer but also in other conditions such as endometriosis. In our patients with endometriotic ovarian cysts serum CA125 levels were significantly higher than in women with other benign ovarian tumors ( $59 \pm 16$ vs. $15 \pm 6 \mathrm{U} / \mathrm{ml}, p<0.05$ ) (Figure 2). All analyzed chemokines - CX3CL1 (fractalkine), CXCL1 (GRO- $\alpha$ ), CXCL12 (SDF-1) had similar serum titers in patients with endometriotic vs. other benign ovarian cysts (Figure 2).

\section{Discussion}

Inflammation is an essential component of the tumor microenvironment of ovarian cancer. The chemokines and cytokines released by cancer and stromal cells are the mediators of inflammation. Chemokines are primarily responsible for support of tumor cell survival and proliferation as well as for recruitment of immune cells from blood to the tumor tissue. They also have a strong pro-angiogenic effect required for tumor growth and progression. The presence of cytokines and chemokines in cancer patients is not connected with the tumor microenvironment only; they are also present in the circulation, since they are secreted into the surrounding tumor blood vessels [2, 3]. Some reports indicate that circulating chemokines could be considered as useful cancer prognostic markers [27, 28]. Therefore, in this study we tested the usefulness of selected chemokines in the preoperative diagnosis of ovarian tumor.
The available data on CX3CL1 involvement in ovarian tumors are extremely limited. Published papers have reported that specimens of both ovarian carcinoma and benign tumors expressed CX3CL1 and its receptor [7, 8, 13]. However, no data on the serum level of this chemokine in patients suffering from ovarian cancer or benign ovarian tumors have been reported. Our results clearly showed that CX3CL1 is elevated in sera of ovarian cancer patients in comparison to benign ovarian tumors, which points to its role in cancer development and/or progression. A higher level of CX3CL1 was also found in the ascites of patients with ovarian cancer than in peritoneal fluid of women with benign gynecologic diseases [29]. One of the proposed explanations of the involvement of CX3CL1 in ovarian cancer development is its high ability to induce cell proliferation via CX$3 C R 1$, which was well documented in the in vitro model of human epithelial ovarian carcinoma cell lines $[7,29]$. Thus, a possible role of CX3CL1 in ovarian carcinogenesis was also proposed by Kim et al. [29], who found that normal ovarian surface epithelium was negative for CX3CR1, while this specific receptor was highly expressed in epithelial ovarian carcinoma, which enables the response of cancer cells to circulating CX3CL1. Furthermore, some authors also found that CX3CL1 presence in malignant ovarian tissue positively correlated with levels of Ki-67 and GILZ, two markers of proliferation in malignant ovarian epithelial cells [7]. Involvement of CX3CL1 in cancer progression is also connected with its ability to induce cell mo- 
A

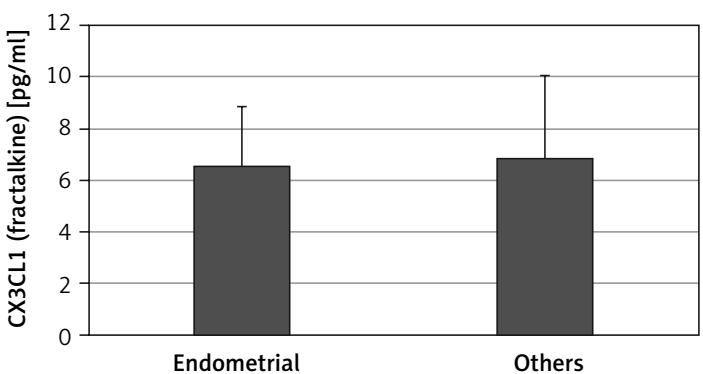

C

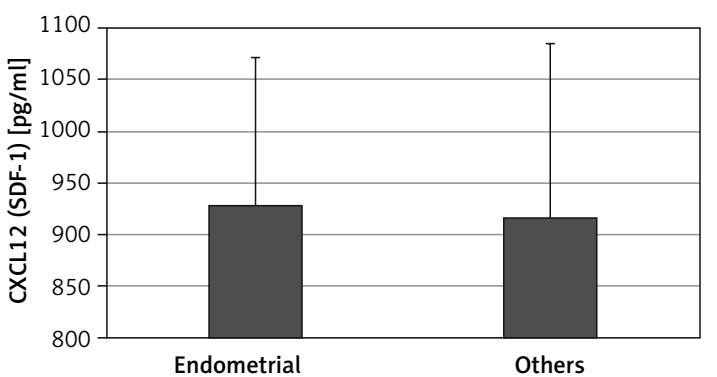

B

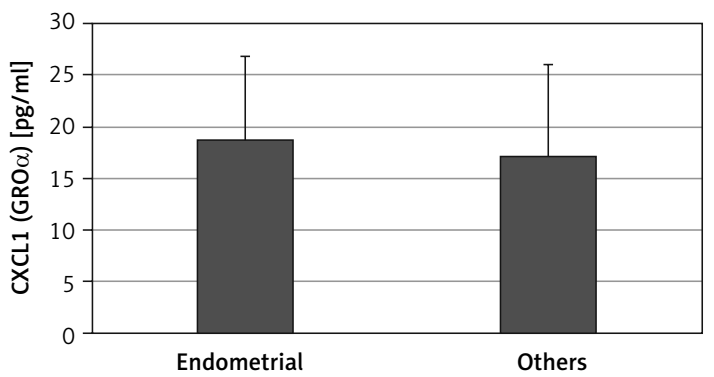

D

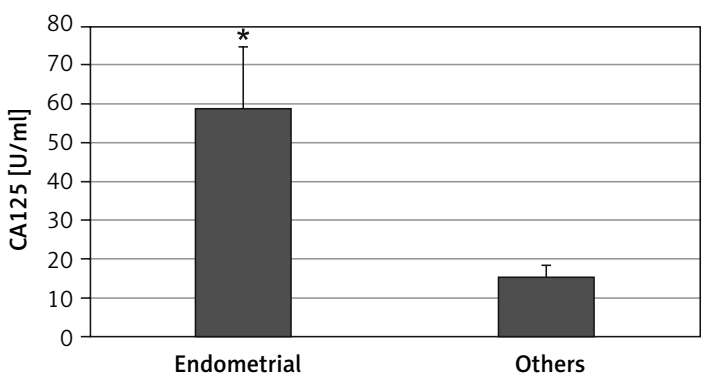

Figure 2. Serum concentrations of selected chemokines and CA125 in patients with benign ovarian tumors. Data are presented as means $\mathrm{pg} / \mathrm{ml} \pm \mathrm{SD}(\mathrm{CA} 125$ as means $\mathrm{U} / \mathrm{ml} \pm \mathrm{SEM})$. Statistical significance: *endometrial ovarian cysts vs. other benign ovarian tumors, $p \leq 0.05$

tility. The proinvasive and promigratory character of CX3CL1 has been demonstrated against the human breast cancer cell line MDA-MB-231 [30] and ovarian cancer cell lines SK-OV-3 and Caov-3 [29] among others. Involvement of CX3CL1 in facilitating epithelial ovarian cancer cells to migrate to the peritoneum and to adhere to the peritoneal wall was also documented [13].

We also observed that the serum level of CXCL1 was significantly higher in the ovarian cancer patients than in those with benign tumors. The elevated concentration of CXCL1 in sera of cancer patients in comparison to normal control subjects [31-33] or benign tumors [27] was found by others, as well. Thus, CXCL1 could be considered as a helpful serological marker, since it provides $97 \%$ specificity and $92 \%$ sensitivity for discriminating ovarian cancer patients from women with benign pelvic masses, whereas sensitivity and specificity of CA125 were significantly lower [27]. A high level of CXCL1 was also found in ascites and tumor tissue of ovarian cancer patients [32, 33]. Moreover, data presented by Couderc et al. [34] clearly showed that the concentration of CXCL1 in the sera of patients with ovarian adenocarcinoma resistant to platinum was higher than in patients with platinum-sensitive tumor. Therefore, CXCL1 was considered as a predictor of ovarian cancer response to chemotherapy. Similarly to our study, it was also reported that the serum level of CXCL1 was not increased in endometriosis patients [35]. It is known that CXCL1 exerts multiple effects directly on the cancer cells as well as on stromal cells such as fibroblasts and immune cells promoting tumor development, progression, and metastasis [2]. It strongly induces the proliferation of tumor cells, as was clearly shown in the studies with various ovarian cancer cell lines overexpressing or lacking CXCL1 or treated with exogenously added chemokine [36]. CXCL1-induced cell proliferation was dependent on its effect on the intracellular signaling pathway (mitogen activated protein kinase, MAPK) involved in cell division and nuclear factor $\kappa B$ responsible for cell survival $[37,38]$. Meanwhile, silencing of CXCL1 in ovarian cancer cells resulted in inhibition of cell growth, invasiveness and aggressiveness $[36,38]$. The pro-metastatic character of CXCL1 was well documented by studies by Yung et al. [38], who found that this chemokine and interleukin 8 are the predominant factors present in the cancerous omentum and they allow cancer cell seeding and growth in the peritoneal cavity. Moreover, CXCL1 was reported to stimulate fibroblast senescence to reprogram the stromal microenvironment, which in turn promoted the malignant transformation of epithelial cells [32]. The importance of CXCL1 in tumor progression may also be connected to its influence on immune cells. Among others, this chemokine controls migration and infiltration of neutrophils to the site of tumor $[2,17]$. Tumor-associated neutrophils are directly implicated in the invasion and metastasis of cancer cells, mainly through remodeling of the basal membrane barrier and enhancement of angiogenesis [39, 40]. 
Thus, CX3CL1 and CXCL1 were both elevated in sera of ovarian cancer patients when compared to women with benign ovarian tumors. Moreover, these chemokines' serum levels did not differ in women with endometriotic vs. other benign cysts. Thus, they might be useful in preoperative differential diagnosis of ovarian tumors, especially considering their behavior in patients with endometriosis. Actually, application of HE4 into the diagnostic protocol of ovarian tumors made it possible to verify the majority of false positive results of CA125 in patients suffering from endometriosis [41]. According to available data, levels of CA125 are elevated in approximately $67 \%$ of cases of endometriosis while HE4 levels are increased only in $3 \%$ of them [42]. Nevertheless, still some endometriomas are preoperatively suspected of potential malignancy. In patients with benign ovarian tumors CA125 and HE4 are elevated in $29 \%$ and $8 \%$ of cases, respectively. False positive results of CA125 and HE4 occur respectively in $20 \%$ and $8 \%$ of serous tumors, $18 \%$ and $13 \%$ of mucinous benign tumors, $26 \%$ and $8 \%$ of fibromas, and $37 \%$ and $10 \%$ of inflammatory lesions [42]. Consequently, despite improvement of the diagnostic protocol after implementation of HE4, before surgical treatment some benign ovarian tumors are constantly considered as malignant. Therefore, discovery and application of new biomarkers might be needed to increase the specificity of preoperative diagnosis of ovarian tumors.

Furthermore, the present diagnostic protocol of ovarian cancer still lacks sensitivity. Sensitivity of CA125 is about $80 \%$, which means that $20 \%$ of patients suffering from ovarian cancer have CA125 levels within the normal range [43]. HE4 levels have been reported to be elevated in over a half of ovarian cancer patients with CA125 values not increased [44]. Despite this fact, overall sensitivity of HE4 is about $74 \%$ [43]. Sensitivity of Risk of Ovarian Malignancy Algorithm (ROMA), which is a diagnostic index combining CA125 and $\mathrm{HE} 4$ levels to evaluate the risk of ovarian cancer, is estimated at about 87\% [43].

Another drawback of CA125 is its low sensitivity in detection of the early stage of ovarian cancer. Among other available biomarkers, HE4 has been reported to be the most sensitive ( $46.2 \%$ sensitivity at $90 \%$ specificity) [44]. Thus, efficacy of ROMA in diagnosis of early stage ovarian cancer is limited $[45,46]$. Consequently, as far as diagnosis of early ovarian cancer is concerned, sensitivity of any biomarker or combination of markers seems to be rather unsatisfactory at the moment.

Moreover, neither CA125 nor HE4 is specific for all histopathological types of ovarian cancer. Although CA125 is overexpressed in most serous ovarian cancers, its levels remain within the normal range in about $70 \%$ of patients suffering from mucinous, clear-cell and endometrioid ovarian cancer [47]. HE4 is overexpressed in the vast majority of serous and endometrioid, over a half of clear-cell and very rarely in mucinous ovarian cancers [48, 49]. According to these data, both CA125 and HE4 are barely useful in diagnosis of less frequent types of ovarian cancer. Thus, a lot of investigators are still looking for new biomarkers and prognostic factors for ovarian cancer $[2,4,24,50,51]$.

In conclusion, our results showed that CX3CL1 and CXCL1 are elevated in sera of ovarian cancer patients, which points to their role in cancer development. As we discussed, present diagnostic protocols of ovarian cancer need improvement regarding both sensitivity and specificity, which might be achieved by application of new biomarkers. Serum levels of CX3CL1 and CXCL1 might be useful in preoperative differential diagnosis of ovarian tumors, especially as they were not elevated in cases of endometriosis.

\section{Acknowledgments}

The study was partly financed from the funds of the Ministry of Science and Higher Education of Poland for conducting a statutory research task of Polish Mother's Memorial Hospital - Research Institute in Lodz (Research task number: 2016/ VII/32-SZB).

\section{Conflict of interest}

The authors declare no conflict of interest.

\section{References}

1. Sokol CL, Luster AD. The chemokine system in innate immunity. Cold Spring Harb Perspect Biol 2015; 7: a016303.

2. Rainczuk A, Rao J, Gathercole J, Stephens AN. The emerging role of CXC chemokines in epithelial ovarian cancer. Reproduction 2012; 144: 303-17.

3. Chow MT, Luster AD. Chemokines in cancer. Cancer Immunol Res 2014; 2: 1125-31.

4. Barbieri F, Bajetto A, Florio T. Role of chemokine network in the development and progression of ovarian cancer: a potential novel pharmacological target. J Oncol 2010; 2010: 1-15.

5. Bazan JF, Bacon KB, Hardiman G, et al. A new class of membrane-bound chemokine with a $\mathrm{CX} 3 \mathrm{C}$ motif. Nature 1997; 385: 640-4.

6. Zlotnik A, Yoshie O. Chemokines: a new classification system and their role in immunity. Immunity 2000; 12 : 121-7.

7. Gaudin F, Nasreddine S, Donnadieu AC, et al. Identification of the chemokine CX3CL1 as a new regulator of malignant cell proliferation in epithelial ovarian cancer. PLoS One 2011; 6: e21546.

8. Gurler H, Macias V, Kajdacsy-Balla A, Barbolina M. Examination of the fractalkine and fractalkine receptor expression in fallopian adenocarcinoma reveals differenc- 
es when compared to ovarian carcinoma. Biomolecules 2015; 5: 3438-47.

9. Jamieson WL, Shimizu S, D’Ambrosio JA, Meucci O, Fatatis A. CX3CR1 is expressed by prostate epithelial cells and androgens regulate the levels of $\mathrm{c} \times 3 \mathrm{cl} 1 /$ fractalkine in the bone marrow: potential role in prostate cancer bone tropism. Cancer Res 2008; 68: 1715-22.

10. Jamieson-Gladney WL, Zhang Y, Fong AM, Meucci O, Fatatis A. The chemokine receptor CX3CR1 is directly involved in the arrest of breast cancer cells to the skeleton. Breast Cancer Res 2011; 13: R91.

11. Marchesi F, Piemonti L, Fedele G, et al. The chemokine receptor CX3CR1 is involved in the neural tropism and malignant behavior of pancreatic ductal adenocarcinoma. Cancer Res 2008; 68: 9060-9.

12. Shulby SA, Dolloff NG, Stearns ME, Meucci O, Fatatis A. CX3CR1-fractalkine expression regulates cellular mechanisms involved in adhesion, migration, and survival of human prostate cancer cells. Cancer Res 2004; 64: 4693-8.

13. Gurler Main H, Xie J, Muralidhar GG, et al. Emergent role of the fractalkine axis in dissemination of peritoneal metastasis from epithelial ovarian carcinoma. Oncogene 2017; 36: 3025-36.

14. Meng $W$, Xue $S$, Chen Y. The role of $C X C L 12$ in tumor microenvironment. Gene 2018; 641: 105-10.

15. Domanska UM, Kruizinga RC, Nagengast WB, et al. A review on CXCR4/CXCL12 axis in oncology: no place to hide. Eur J Cancer 2013; 49: 219-30.

16. Liu Y, Ren CC, Yang L, Xu YM, Chen YN. Role of CXCL12-CXCR4 axis in ovarian cancer metastasis and CXCL12-CXCR4 blockade with AMD3100 suppresses tumor cell migration and invasion in vitro. J Cell Physiol 2019; 234: 3897-909.

17. Girbl T, Lenn T, Perez L, et al. Distinct compartmentalization of the chemokines CXCL1 and CXCL2 and the atypical receptor ACKR1 determine discrete stages of neutrophil diapedesis. Immunity 2018; 49: 1062-76.

18. Hembruff SL, Cheng N. Chemokine signaling in cancer: implications on the tumor microenvironment and therapeutic targeting. Cancer Ther 2009; 7: 254-67.

19. Agarwal A, Tressel SL, Kaimal R, et al. Identification of a metalloprotease-chemokine signaling system in the ovarian cancer microenvironment: implications for antiangiogenic therapy. Cancer Res 2010; 70: 5880-90.

20. Osuala KO, Sloane BF. Many roles of CCL20: emphasis on breast cancer. Postdoc J a J Postdr Res Postdr Aff 2014; 2: 7-16

21. Ignacio RMC, Kabir SM, Lee ES, Adunyah SE, Son DS. NF-KB-mediated CCL20 reigns dominantly in CXCR2-driven ovarian cancer progression. PLoS One 2016; 11: e0164189.

22. Son DS, Kabir SM, Dong Y, Lee E, Adunyah SE. Characteristics of chemokine signatures elicited by EGF and TNF in ovarian cancer cells. J Inflamm 2013; 10: 25.

23. Chang SH, Dong C. Signaling of interleukin-17 family cytokines in immunity and inflammation. Cell Signal 2011; 23: 1069-75.

24. Muinao T, Deka Boruah HP, Pal M. Diagnostic and prognostic biomarkers in ovarian cancer and the potential roles of cancer stem cells - an updated review. Exp Cell Res 2018; 362: 1-10.

25. Prat J. FIGO's staging classification for cancer of the ovary, fallopian tube, and peritoneum: abridged republication. J Gynecol Oncol 2015; 26: 87-9.

26. Meinhold-Heerlein I, Fotopoulou C, Harter P, et al. The new WHO classification of ovarian, fallopian tube, and primary peritoneal cancer and its clinical implications. Arch Gynecol Obstet 2016; 293: 695-700.

27. Wang O, Li D, Zhang W, Tang B, Li OO, Li L. Evaluation of proteomics-identified CCL18 and CXCL1 as circulating tumor markers for differential diagnosis between ovarian carcinomas and benign pelvic masses. Int J Biol Markers 2011; 26: 262-73.

28. Mlynska A, Salciuniene G, Zilionyte K, et al. Chemokine profiling in serum from patients with ovarian cancer reveals candidate biomarkers for recurrence and immune infiltration. Oncol Rep 2019; 41: 1238-52.

29. Kim M, Rooper L, Xie J, Kajdacsy-Balla AA, Barbolina MV. Fractalkine receptor CX3CR1 is expressed in epithelial ovarian carcinoma cells and required for motility and adhesion to peritoneal mesothelial cells. Mol Cancer Res 2012; 10: 11-24.

30. Liang Y, Yi L, Liu P, et al. CX3CL1 involves in breast cancer metastasizing to the spine via the Src/FAK signaling pathway. J Cancer 2018; 9: 3603-12.

31. Nishikawa R, Suzumori N, Nishiyama T, Nishikawa $H$, Arakawa A, Sugiura-Ogasawara M. Clinical significance of serum growth-regulated oncogene $\alpha(G R O \alpha)$ in patients with gynecological cancer. Eur J Gynaecol Oncol 2012; 33: 138-41.

32. Yang G, Rosen DG, Zhang Z, et al. The chemokine growth-regulated oncogene 1 (Gro-1) links RAS signaling to the senescence of stromal fibroblasts and ovarian tumorigenesis. Proc Natl Acad Sci 2006; 103: 16472-7.

33. Lee Z, Swaby RF, Liang Y, et al. Lysophosphatidic acid is a major regulator of growth-regulated oncogene $\alpha$ in ovarian cancer. Cancer Res 2006; 66: 2740-8.

34. Couderc B, Le Naour A, Prat M, et al. Mesenchymal stromal cells release $C X C L 1 / 2 / 8$ and induce chemoresistance and macrophage polarization. bioRxiv 2018: 482513.

35. Pateisky P, Pils D, Kuessel L, et al. The serum levels of the soluble factors SCD4OL and CXCL1 are not indicative of endometriosis. Biomed Res Int 2016; 2016: 1-8.

36. Kavandi L, Collier MA, Nguyen H, Syed V. Progesterone and calcitriol attenuate inflammatory cytokines CXCL1 and CXCL2 in ovarian and endometrial cancer cells. J Cell Biochem 2012; 113: 3143-52.

37. Bolitho C, Hahn MA, Baxter RC, Marsh DJ. The chemokine CXCL1 induces proliferation in epithelial ovarian cancer cells by transactivation of the epidermal growth factor receptor. Endocr Relat Cancer 2010; 17: 929-40.

38. Yung MMH, Tang HWM, Cai PCH, et al. GRO- $\alpha$ and IL- 8 enhance ovarian cancer metastatic potential via the CXCR2-mediated TAK1/NFKB signaling cascade. Theranostics 2018; 8: 1270-85.

39. Fridlender ZG, Albelda SM. Tumor-associated neutrophils: friend or foe? Carcinogenesis 2012; 33: 949-55.

40. Klink M, Sulowska Z. Polymorphonuclear neutrophils and tumors: friend or foe? In: Interaction of Immune and Cancer Cells. Klink M (ed.). Springer, Vienna 2014; 123-41.

41. Huhtinen K, Suvitie P, Hiissa J, et al. Serum HE4 concentration differentiates malignant ovarian tumours from ovarian endometriotic cysts. Br J Cancer 2009; 100: 1315-9.

42. Moore RG, Miller MC, Steinhoff MM, et al. Serum HE4 levels are less frequently elevated than CA125 in women with benign gynecologic disorders. Am J Obstet Gynecol 2012; 206: 351.e1-8.

43. Lin J, Qin J, Sangvatanakul V. Human epididymis protein 4 for differential diagnosis between benign gynecologic disease and ovarian cancer: a systematic review and meta-analysis. Eur J Obstet Gynecol Reprod Biol 2013; 167: 81-5. 
44. Moore RG, Brown AK, Miller MC, et al. The use of multiple novel tumor biomarkers for the detection of ovarian carcinoma in patients with a pelvic mass. Gynecol Oncol 2008; 108: 402-8.

45. Moore RG, Jabre-Raughley M, Brown AK, et al. Comparison of a novel multiple marker assay vs the risk of malignancy index for the prediction of epithelial ovarian cancer in patients with a pelvic mass. Am J Obstet Gynecol 2010; 203: 228.e1-6.

46. Chudecka-Głaz AM. ROMA, an algorithm for ovarian cancer. Clin Chim Acta 2015; 440: 143-51.

47. Mainguené C, Aillet G, Kremer M, Chatal JF. Immunohistochemical study of ovarian tumors using the OC 125 monoclonal antibody as a basis for potential in vivo and in vitro applications. J Nucl Med Allied Sci 1986; 30: 19-22.

48. Drapkin R, von Horsten $\mathrm{HH}$, Lin $\mathrm{Y}$, et al. Human epididymis protein 4 (HE4) is a secreted glycoprotein that is overexpressed by serous and endometrioid ovarian carcinomas. Cancer Res 2005; 65: 2162-9.

49. Galgano MT, Hampton GM, Frierson HF. Comprehensive analysis of HE4 expression in normal and malignant human tissues. Mod Pathol 2006; 19: 847-53.

50. Gu LP, Jin S, Xu RC, et al. Long non-coding RNA PCAT-1 promotes tumor progression by inhibiting miR-129-5p in human ovarian cancer. Arch Med Sci 2019; 15: 513-21.

51. Shao S, Tian J, Zhang H, Wang S. LncRNA myocardial infarction-associated transcript promotes cell proliferation and inhibits cell apoptosis by targeting miR-330-5p in epithelial ovarian cancer cells. Arch Med Sci 2018; 14: 1263-70. 